\title{
Experimental and Simulation-based Investigation of a Velocity Controller Extension on a Ball Screw System
}

\author{
Rico Münster, Michael Walther, Holger Schlegel and Welf-Guntram Drossel \\ Institute of Machine Tools and Production Processes, Faculty of Mechanical Engineering, Technische Universität Chemnitz, \\ Chemnitz 09126, Germany
}

Received: March 11, 2015 / Accepted: March 30, 2015 / Published: April 25, 2015.

\begin{abstract}
The background of this contribution is the enhancement of the achievable accuracy of servo-screw-presses. Therefore, the paper is concerned with the improvement of the dynamic precision for direct driven servo axes which is still restricted by structural vibrations. For this purpose, a ball screw test rig was analyzed for which the standard cascade control structure was extended by an additional velocity feedback. This structural extension has the potential to improve the controller performance significantly due to a better damping of low frequent vibrations. Furthermore, a parametric dynamic model for the control structure was derived to investigate the effects of the controller extension. For this analysis, the influences of the used tuning factors and filters is discussed in the frequency domain based on bode plots. The results of these cognitions are transferred to the time domain and illustrated by step responses. In addition, an evaluation by the criterion of the IAE (integral of absolute error) and the Prony analysis is carried out. Finally, the results are experimentally verified at the ball screw test rig. The paper closes with the conclusions.
\end{abstract}

Key words: Velocity controller extension, servo-screw-press, advanced position control, integral absolute error, Prony analysis.

\section{Introduction}

In recent years, servo-screw-presses achieved a huge importance in forming technology because of their high degree of flexibility. By the use of their nearly free selectable motion characteristics in tool movement they have a considerable potential to beneficially move the process limitations like cycle times, product accuracy and energy consumption. Furthermore, they offer the capability for new production processes, like a pulsing ram movement in which several forming steps can be put together [1].

At present, the position control of servo-screw-presses is based on an indirect feedback [2]. This means that the motor velocity is fed back into the control loop. As a result the elasticity of the mechanical structure and other disturbing effects are not considered in the feedback control. To fully take

Corresponding author: Holger Schlegel, Dr.-Ing., research fields: control and feedback control technologies. E-mail: holger.schlegel@mb.tu-chemnitz.de. advantage of the possibilities, these effects have to be included in the control structure by utilizing a direct position signal. In this case, this is the ram position as shown in Fig. 1. Yet, this leads to a limitation in control performance because of low frequent structural vibrations. Preliminary investigation on the servo-screw-press showed, that there exist several eigenfrequencies in the lower frequency range up to $100 \mathrm{~Hz}$ [1].

A possible approach to compensate this limitation is

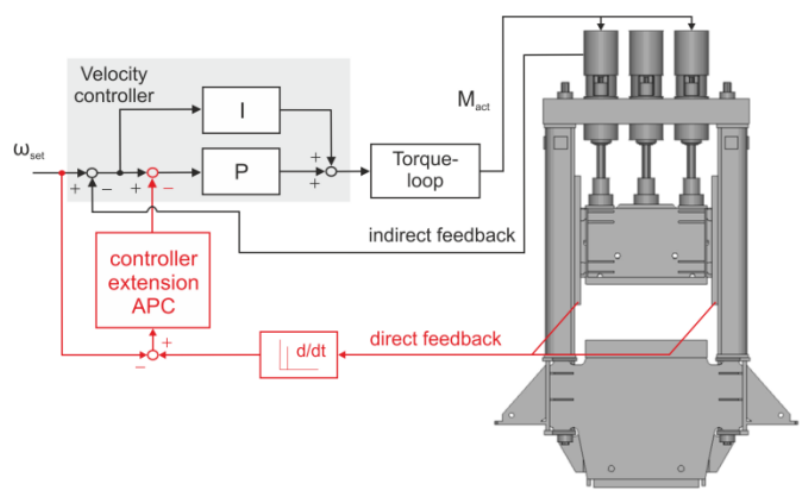

Fig. 1 Control structure of a servo-screw-press. 
the extension of the velocity controller which is object of current research [2], 3] and will be investigated in this paper by analyzing a ball screw system which is comparable to the direct driven servo axes of the servo-screw-press. Therefore, the mechanic structure of the controlled system and a standard PI (proportional-integral) velocity control system is modeled.

After commissioning the standard controller cascade, the additional velocity controller extension can be implemented. The analysis of this extension is investigated in the frequency and time domain. Finally, an evaluation of the results is presented by the criterion of the IAE (integral of absolute error) and the Prony analysis. Based on these results, transferability from the ball screw to the servo press can be derived and the benefits of the controller extension can be estimated.

\section{Velocity Controller Extension}

For the velocity control of axes with low frequent vibration modes, the standard PI velocity controller, even with an appropriate setting, is insufficient because of a comparatively small damping. Therefore the extension of the velocity controller with an additional feedback of the load side measured position will be investigated. With this approach, a significantly better damping of structural vibrations can be achieved. However, the need of additional measurement devices, an even more complex control structure and comparatively high commissioning efforts make this velocity controller extension unattractive for most control system users. Nevertheless, one of the leading control system manufacturers offers such an extension called APC (advanced position control) for servo axes. The option is integrated in their servo drive system [4]. It offers the advantage that the standard PI cascade structure remains the same.

In addition to the standard velocity control loop, the load side measured position is fed back as first derivation for the velocity and second derivation for the acceleration path, as shown in Fig. 2. Therefore, the velocity path comprises the difference between the load velocity and the setpoint value, multiplied by a tuning factor, denoted as gain $K_{\mathrm{APC} 1} / K_{\mathrm{APC} 2}$. The acceleration path feeds back the load acceleration multiplied by a derivative time constant $T_{\mathrm{APC} 1} / T_{\mathrm{APC} 2}$. Subsequently, the velocity and acceleration paths are summed up and optionally filtered. For this filtering, a freely adjustable filter structure of second order is provided by the servo drive system.

It can be used to specifically damp or gain respective frequencies [5].

$$
G_{\text {filter }}(s)=\frac{\frac{1}{\left(2 \pi \cdot f_{n_{-} \text {num }}\right)^{2}} \cdot s^{2}+\frac{2 \cdot D_{\text {num }}}{2 \pi \cdot f_{n_{-} \text {num }}} \cdot s+1}{\frac{1}{\left(2 \pi \cdot f_{n_{-} \text {den }}\right)^{2}} \cdot s^{2}+\frac{2 \cdot D_{\text {den }}}{2 \pi \cdot f_{n_{-} \text {den }}} \cdot s+1}
$$

This double feedback is provided for filtering up to two relevant frequencies which are summed up and fed back into the velocity control loop [6]. After this double feedback, a low pass filter can be used for reducing, e.g., quantization noise.

The advantage of the APC is the consideration of the structural behavior by using the relevant position signal at the load side in addition to the indirect motor signal. With this direct processing of structural vibrations, a better dynamic controller performance can be achieved.

\section{Modeling}

For the investigation of the velocity controller extension, a ball screw test rig was selected. It consists of a synchronous servo motor, a torque measurement shaft, a clutch, a ball screw spindle, a slide with additional mass and a brake with counter bearing. The individual components are shown in Fig. 3.

For modeling the controlled system, the mechanical structure has to be identified first. Therefore, the frequency response of the ball screw test rig was measured (Fig. 4). Since five relevant natural 


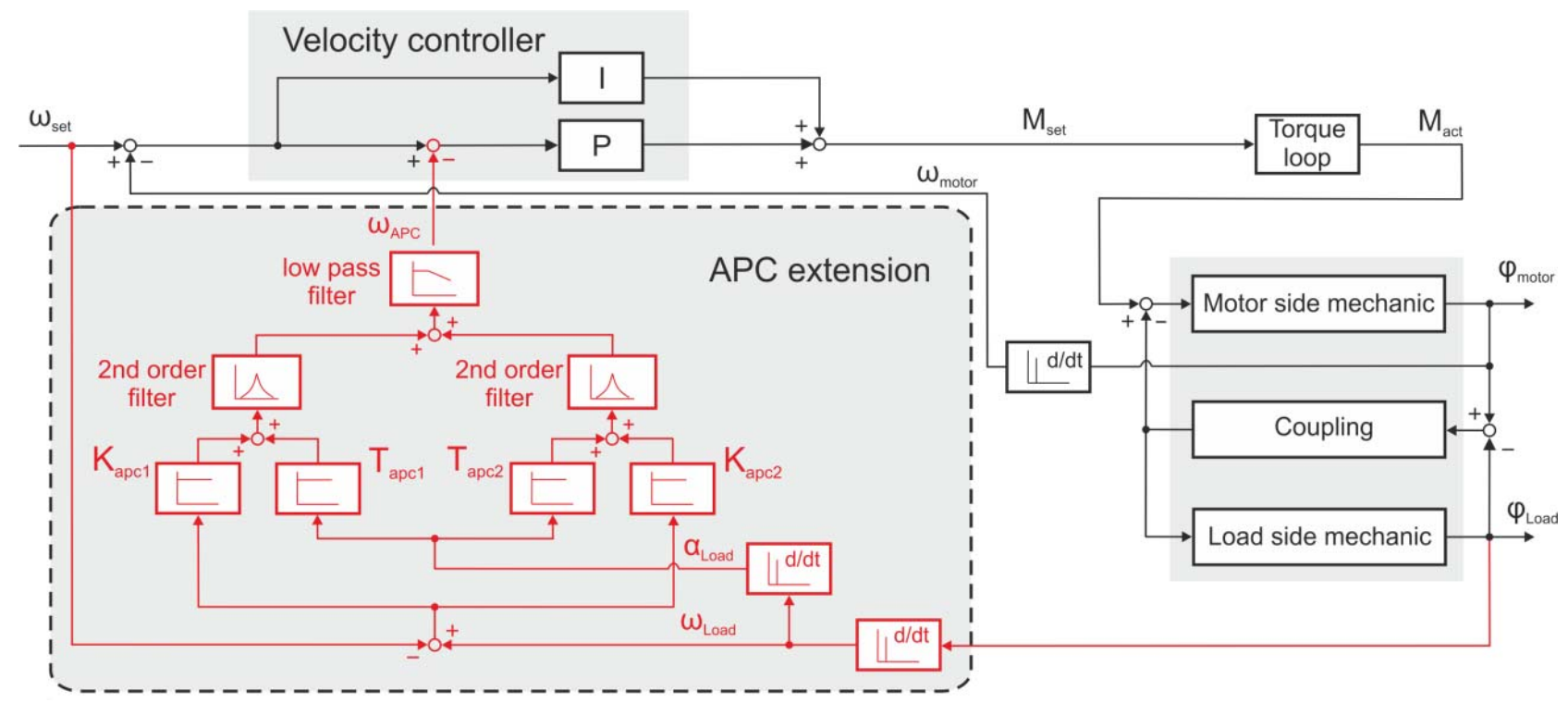

Fig. 2 Block diagram of the APC option, APC structure from Ref. [6].

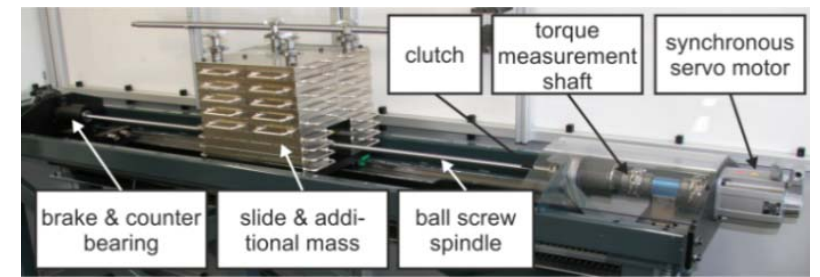

Fig. 3 Ball screw test rig.

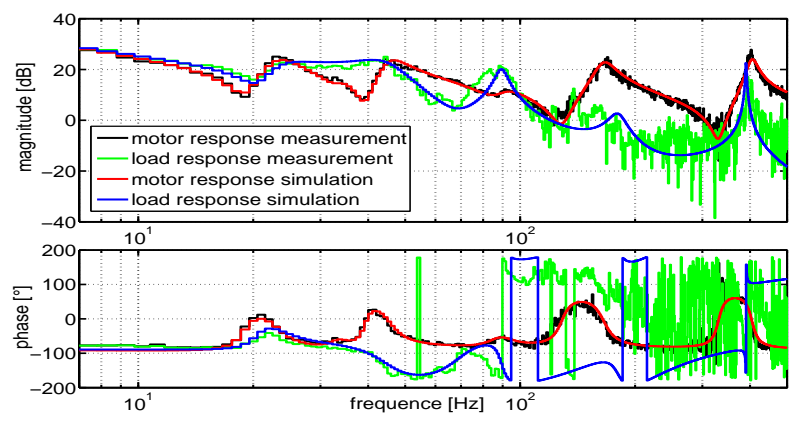

Fig. 4 Frequency response for controlled system.

frequencies in the frequency range up to $500 \mathrm{~Hz}$ can be identified, it would be necessary to use a six-mass-system. Because it is very difficult to derive such a model out of the mechanic properties, we abstain from modeling with spring-mass elements. For the detailed simulative reconstruction of the measured frequency response, we used general transfer elements instead. As a result, the controlled system comprises the overall mass moment of inertia of the mechanics as well as the individual sub-oscillators [7, 8]. For the transfer function of the mechanical system, there has to be made a distinction between motor and load side which differ by their individual resonances respectively, as visible in Eq. (2).

$$
\begin{aligned}
& G_{s}(s)=\frac{1}{J_{t o t} \cdot s} \cdot \frac{\frac{1}{\omega_{\text {anti_1 }}{ }^{2}} \cdot s^{2}+\frac{2 \cdot D_{\text {num_ } 1}}{\omega_{\text {ant__ } 1}} \cdot s+1}{\frac{1}{\omega_{\text {res_1 }}{ }^{2}} \cdot s^{2}+\frac{2 \cdot D_{\text {den_1 }}}{\omega_{\text {res } 1}} \cdot s+1} . \\
& \ldots \cdot \frac{\frac{1}{\omega_{\text {anti_ } 5}{ }^{2}} \cdot s^{2}+\frac{2 \cdot D_{\text {num }_{-} 5}}{\omega_{\text {anti_ } 5}} \cdot s+1}{\frac{1}{\omega_{\text {res_ } 5}{ }^{2}} \cdot s^{2}+\frac{2 \cdot D_{\text {den }_{-} 5}}{\omega_{\text {res } 5} 5} \cdot s+1}
\end{aligned}
$$

The total mass moment of inertia was taken from the construction data and has an amount of $J_{t o t}=8.74$ $\times 10^{-3} \mathrm{kgm}^{2}$. The resonant and antiresonant frequencies were derived from the results of the measurements, whereas the numerator- and denumerator-dampings were approximated empirically during modeling (Table 1).

With the determined mechanical system, the velocity controller can be designed. The integral time constant $T_{N}=10.057 \mathrm{~m} / \mathrm{s}$ of the velocity controller was taken from the automatic controller commissioning of the drive system. Because the proportional gain $K_{P}$ $=2.864 \mathrm{Nms} / \mathrm{rad}$ of the automatic commissioning 
Table 1 Resonances and antiresonances for motor and load side mechanic.

\begin{tabular}{|c|c|c|c|}
\hline \multicolumn{2}{|c|}{ Motor side mechanic } & \multicolumn{2}{|c|}{ Load side mechanic } \\
\hline $\begin{array}{l}\text { Antiresonance } \\
(\mathrm{Hz})\end{array}$ & $\begin{array}{l}\text { Resonance } \\
(\mathrm{Hz})\end{array}$ & $\begin{array}{l}\text { Antiresonance } \\
(\mathrm{Hz})\end{array}$ & $\begin{array}{l}\text { Resonance } \\
(\mathrm{Hz})\end{array}$ \\
\hline 18.5 & 22.5 & 20.5 & 22.5 \\
\hline 32.3 & 33.3 & - & - \\
\hline 39 & 45 & 32.2 & 44 \\
\hline 86.0 & 90.0 & 68 & 89 \\
\hline 128.0 & 165 & 163 & 180 \\
\hline 330 & 404 & 205 & 390 \\
\hline
\end{tabular}

was too high and caused too high overshot, it was reduced to $K_{P}=1.0 \mathrm{Nms} / \mathrm{rad}$. Another reason for this adjustment is the larger required phase reserve for the additional use of the APC feedback.

\section{Simulation}

After commissioning the velocity controller, the APC option can be implemented. In the model, the individual velocity and acceleration paths and their filters are investigated.

At first, the velocity feedback with the tuning factor $K_{\mathrm{APC} 1}=1.0$ is presented in Fig. 5 . It can be noticed that the first dominating frequency at $15 \mathrm{~Hz}$ and also partly the second frequency at $30 \mathrm{~Hz}$ is damped by this feedback path. However, this results in an increase of amplitude for the following dominating frequencies at $45 \mathrm{~Hz}, 93 \mathrm{~Hz}$ and $185 \mathrm{~Hz}$. It appears that the APC proportional velocity feedback only achieves a damping of lower frequencies if the following higher frequencies are amplified at the same time.

In the next step the effects of the APC acceleration path are shown in the frequency response in Fig. 6. In this case, the derivative time constant $T_{\mathrm{APC} 1}=2 \mathrm{~m} / \mathrm{s}$ causes nearly no damping of the first frequency, but a significant damping for the second and third frequencies at $30 \mathrm{~Hz}$ and $45 \mathrm{~Hz}$. The effect of amplifying the following frequencies is virtually negligible.

In Fig. 7, the velocity as well as the acceleration path is used. Here, it is clearly visible that both effects appear superimposed.

The velocity feedback damps the first frequency while both effects lead to a damping of the second and third frequency. In addition, the strong amplification of the frequency at $93 \mathrm{~Hz}$ can considerably be damped by the use of the acceleration path.

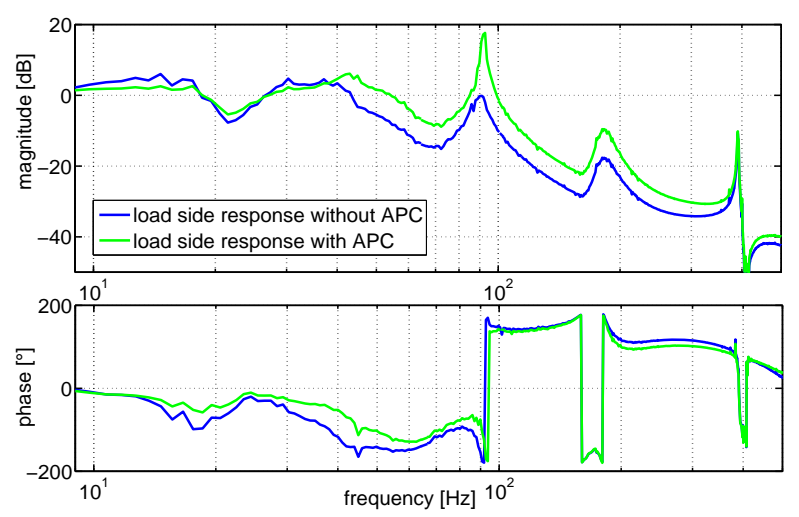

Fig. 5 Closed loop load side frequency response for $K_{\mathrm{APC}}$ gain.

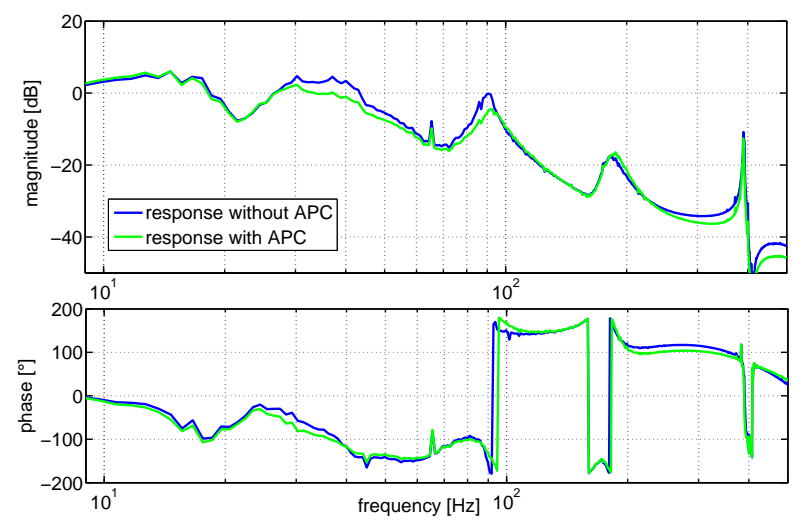

Fig. 6 Closed loop load side frequency response for derivative time constant $T_{\mathrm{APC}}$.

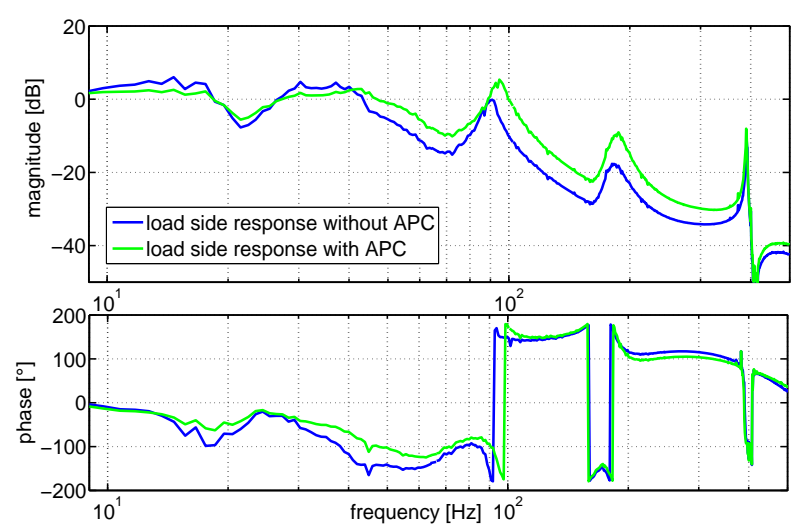

Fig. 7 Closed loop load side frequency response for $K_{\mathrm{APC}}$ $\& T_{\mathrm{APC}}$. 


\section{Evaluation}

After investigating the influences of the APC tuning factors, a suitable parameterization has to be found. Therefore, a simulation based optimization is performed [9]. For representing the best damping behavior of the system, several optimization criteria were investigated. In this paper, the results of the IAE were chosen, as advised in Ref. [10], where $x_{d}$ is the velocity error signal.

$$
I A E=\int_{0}^{\infty}\left|x_{d}(t)\right| d t
$$

At first, the IAE was measured in a time of $0.5 \mathrm{~s}$ beginning from the step for the standard PI control structure without APC (Table 2). Referring to this, the IAE of the additional APC structure was minimized, based on the variation of the tuning factors $K_{\mathrm{APC} 1}$ and $T_{\text {APC1 }}$ without any filter. The results can be found in Table 3. In this case, the optimal combination of system damping and loss of the velocity controller performance is reached whereby smaller tuning gains lead to lower damping and higher gains lead to greater loss of performance. In addition, the simulation based optimization analyzed the influence of an additionally used filter of second order in the APC feedback.

The optimization showed that by using an additional filter, the tuning factors could be increased even more (Table 4). The determined filter is shown in Fig. 8. It can be seen that the filter frequency response is higher than the respective frequency of the response which is damped. This compromise results from the fact that the lower frequencies must not be affected through the filter. Therefore, the filter additionally damps the higher frequencies and raises the phase.

Comparing the optimal APC settings with the standard control structure, a significantly smaller IAE can be reached for the load velocity.

The APC setting without any filter reached a more than two times smaller IAE whereby the APC setting with an additional filter even reached a three times smaller IAE. At the same time, the IAE of the motor nearly stayed the same. For the APC setting without
Table 2 Integral of absolute error.

\begin{tabular}{lll}
\hline & IAE & Parameters \\
\hline IAE load without APC & 155.4 & - \\
IAE load with APC & 64.2 & Table 3 \\
IAE load with APC \& filter & 45.9 & Table 4 \\
IAE motor without APC & 107.6 & - \\
IAE motor with APC & 101.2 & Table 3 \\
IAE motor with APC \& filter & 122.7 & Table 4 \\
\hline
\end{tabular}

Table 3 Optimal setting for APC without filter.

\begin{tabular}{ll}
\hline$K_{\mathrm{APC} 1}(-)$ & 3.07 \\
\hline$T_{\mathrm{APC} 1}(\mathrm{~m} / \mathrm{s})$ & 3.81 \\
\hline
\end{tabular}

Table 4 Optimal setting for APC \& filter.

\begin{tabular}{ll}
\hline$K_{\mathrm{APC} 1}$ & 4.60 \\
\hline$T_{\mathrm{APC} 1}(\mathrm{~m} / \mathrm{s})$ & 7.70 \\
\hline$f_{n \_n u m}(\mathrm{~Hz})$ & 353 \\
\hline$f_{n \_ \text {den }}(\mathrm{Hz})$ & 335 \\
\hline$D_{\text {num }}$ & 0.44 \\
\hline$D_{\text {den }}$ & 0.29 \\
\hline
\end{tabular}

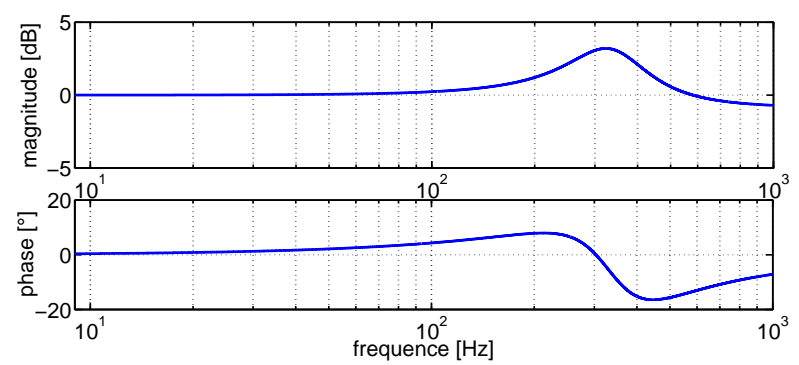

Fig. 8 Additionally used filter in APC feedback.

filter, the IAE could slightly be reduced and for the APC setting with additional filter the IAE slightly increased. This shows that the potential of the APC structure could fully be exploited.

\subsection{Analysis in the Time Domain}

To illustrate the influence of APC, the step responses of the load and motor velocity with and without APC is put in contrast.

It turns out that with the APC option a better damping of the load velocity can be achieved, as shown in Fig. 9. It can be seen that the critical amplitudes of the load velocity, caused by the lower frequent vibrations, can be reduced significantly while higher frequencies oscillate slightly more. By using an additional filter, the damping effect is even stronger, 


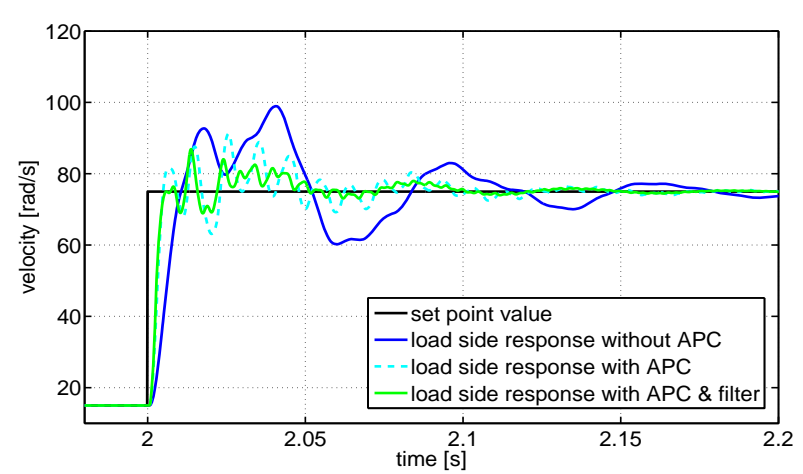

Fig. 9 Step response of load velocity.

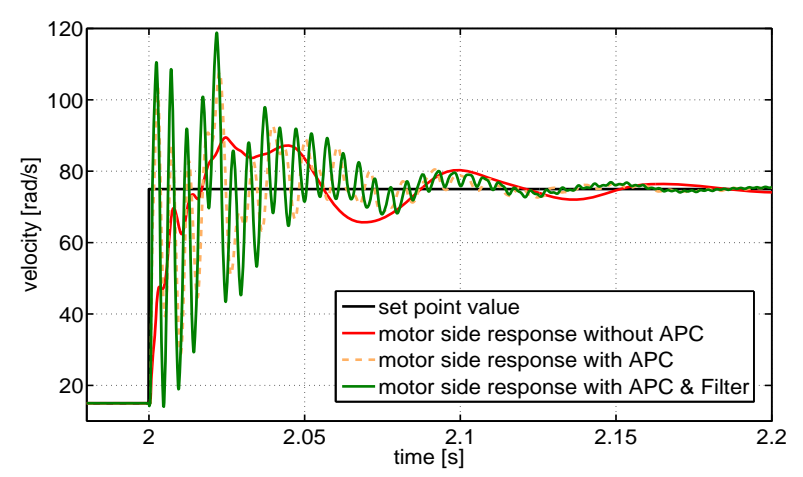

Fig. 10 Step response of motor velocity.

because also the higher frequency can be damped.

The motor velocity in Fig. 10 shows a similar tendency. Again, the low frequent vibrations are damped, which results in a large excitation of higher frequencies. However, this is necessary to enable the damping on the load side.

It can be stated that with the utilization of the APC feedback, a significantly better damping can be realized. It can be seen that the motor counteracts the feedback velocity so that the damping at the load side can be achieved. This results in a considerable increase of the setpoint torque from $M_{\text {set }}=7.3 \mathrm{Nm}$ for the standard control to $M_{\text {set }}=26.4 \mathrm{Nm}$ for APC without filter which is still under the maximum torque of $36 \mathrm{Nm}$ for the used servo motor.

For the APC option with additional filter, the maximum torque would be over the permissible torque. For that reason, a torque limitation at $M_{\text {lim }}=32$ $\mathrm{Nm}$ is used in simulation as well as at the test rig.

\subsection{Prony Analysis}

The Prony Analysis originally is a method of spectral analysis. In comparison to the better known Fourier analysis, some significant differences can be noted. In contrast to the Fourier analysis, the Prony analysis can be determined as a parametric method. It offers the advantage that even comparatively short time series are adequate for useful results [11]. For modeling the examined signal, the Prony analysis uses damped oscillations:

$$
\hat{x}_{n}=\sum_{m=0}^{p} b_{m} \cdot z_{m}^{n}
$$

with

$$
b_{m}=A_{m} \cdot e^{j \Phi_{m}}
$$

and

$$
z_{m}=e^{\left(\gamma_{m}+j \omega_{m}\right) T_{\text {sample }}}
$$

The signal is composed as a sum of a discrete number of damped sinusoids with the amplitudes $A_{m}$, frequencies $\omega_{m}$, phase shifts $\Phi_{m}$ and damping ratios $\gamma_{m}$. The analysis thereby focuses on the minimization of the error between the estimated signal $\hat{x}_{n}$ and the measured signal $x_{n}$. The parameters frequency, amplitude and damping which have to be determined, are direct analytical results and therefore well to evaluate. The mathematical derivation is omitted here [12].

In Table 5, the determined parameters are listed for three settings: standard structure without APC, extended structure with APC and extended structure with APC plus additional filter.

All dominating frequencies including their amplitudes and damping could be identified with the Prony analysis for each setting. Looking at the first two frequencies, the damping effect becomes clearly visible through the decreasing amplitudes. The second relevant frequency is actually moving to higher frequencies from $35 \mathrm{~Hz}$ to $62 \mathrm{~Hz}$. The following four dominating frequencies are getting amplified which is characterized through their increasing amplitudes. Looking at frequency number three, the moving to higher frequencies from $89 \mathrm{~Hz}$ to $110 \mathrm{~Hz}$ can also been seen. 
Table 5 Determined dynamic parameters from Prony analysis for the load side mechanic.

\begin{tabular}{|c|c|c|c|c|}
\hline \multirow{2}{*}{$\begin{array}{l}\text { Number of } \\
\text { frequency }\end{array}$} & \multirow{2}{*}{ Parameter } & \multicolumn{2}{|c|}{ With-out With } & \multirow{2}{*}{$\begin{array}{l}\text { With APC } \\
\text { \& filter }\end{array}$} \\
\hline & & APC & APC & \\
\hline \multirow{3}{*}{1.} & Frequency $(\mathrm{Hz})$ & 14.5 & 14.5 & 14.12 \\
\hline & Amplitude (rad/s) & 26.7 & 10.5 & 3.9 \\
\hline & Damping & 0.12 & 0.22 & 0.20 \\
\hline \multirow{3}{*}{2.} & Frequency (Hz) & 35.5 & 52.3 & 62.1 \\
\hline & Amplitude (rad/s) & 26.8 & 23.3 & 19.0 \\
\hline & Damping & 0.15 & 0.26 & 0.29 \\
\hline \multirow{3}{*}{3.} & Frequency $(\mathrm{Hz})$ & 89.0 & 101.9 & 110.4 \\
\hline & Amplitude (rad/s) & 3.8 & 15.2 & 16.4 \\
\hline & Damping & 0.03 & 0.04 & 0.11 \\
\hline \multirow{3}{*}{4.} & Frequency (Hz) & 182.1 & 188.0 & 197.6 \\
\hline & Amplitude (rad/s) & 0.7 & 3.7 & 4.7 \\
\hline & Damping & 0.05 & 0.03 & 0.03 \\
\hline \multirow{3}{*}{5.} & Frequency (Hz) & 390.0 & 387.8 & 383.4 \\
\hline & Amplitude (rad/s) & 0.1 & 0.3 & 0.7 \\
\hline & Damping & 0.01 & 0.01 & 0.01 \\
\hline
\end{tabular}

\subsection{Analysis in the Frequency Domain}

Considering the frequency response, the damping effect of APC gets even clearer. Fig. 11 shows that with the optimal APC settings, used in the simulation, an optimal damping for the load side amplitude up to $90 \mathrm{~Hz}$ can be achieved. If the APC option is used without an additional filter, the damping of lower frequencies results in an amplification of the following frequencies, especially the dominant frequency at $102 \mathrm{~Hz}$. With the use of the filter, the amplification of the dominant frequency $102 \mathrm{~Hz}$ is reduced significantly without influencing the damping of lower frequencies.

The frequency response of the motor side in Fig. 12 shows a similar tendency. The amplitude is damped up to $45 \mathrm{~Hz}$ while the following frequencies at $60 \mathrm{~Hz}$, $102 \mathrm{~Hz}$ and $185 \mathrm{~Hz}$ are significantly amplified. Like for the load side magnitude, the usage of the feedback filter also damps the critical frequency at $102 \mathrm{~Hz}$ of the motor side. As a result, higher frequencies are gained so that the optimized APC setting with additional filter exploits all the potential of the APC feedback and thus represents the stability limit.

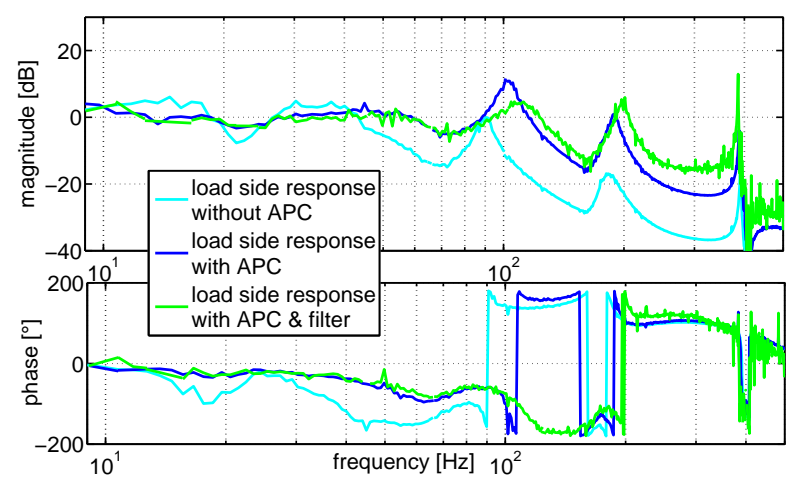

Fig. 11 Optimized frequency response of load velocity.

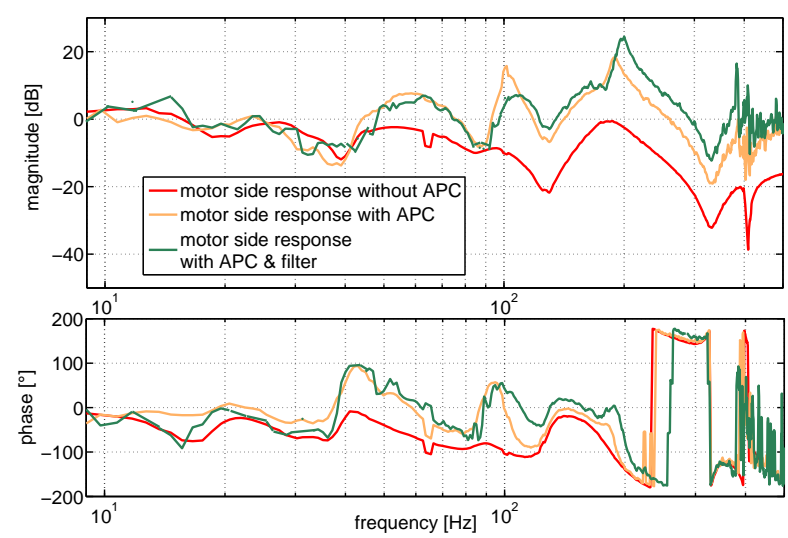

Fig. 12 Optimized frequency response of motor velocity.

Regarding the phase, it can be seen that with the usage of APC, the phase can be raised at the load side as well as at the motor side.

\section{Experimental Verification}

To verify the results of the simulation, the determined parameter settings were now reproduced by means of experiments at the ball screw test rig. At first, the step response of the motor and load side is investigated (Fig. 13). The system responses are considered for the standard structure without APC and the extended structure with APC. For the APC parameterization, the tuning factors $K_{\mathrm{APC}}=0.2$ and $T_{\mathrm{APC}}=0.5 \mathrm{~m} / \mathrm{s}$ were used.

It can be seen that the amplitudes of the velocity from the simulation as well as from the experiment show a good accordance. Only the low frequent vibrations indicate a slightly different curve shape, which can be seen in deviations in the transient response. 


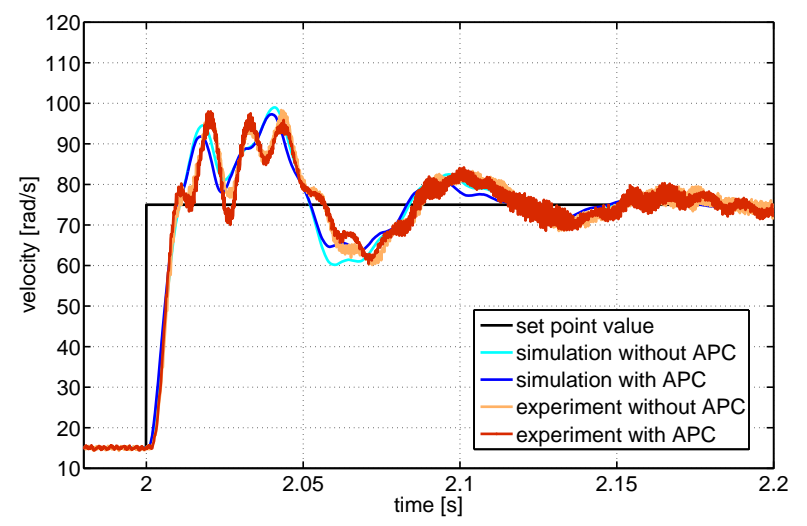

Fig. 13 Step response of simulation and experiment.

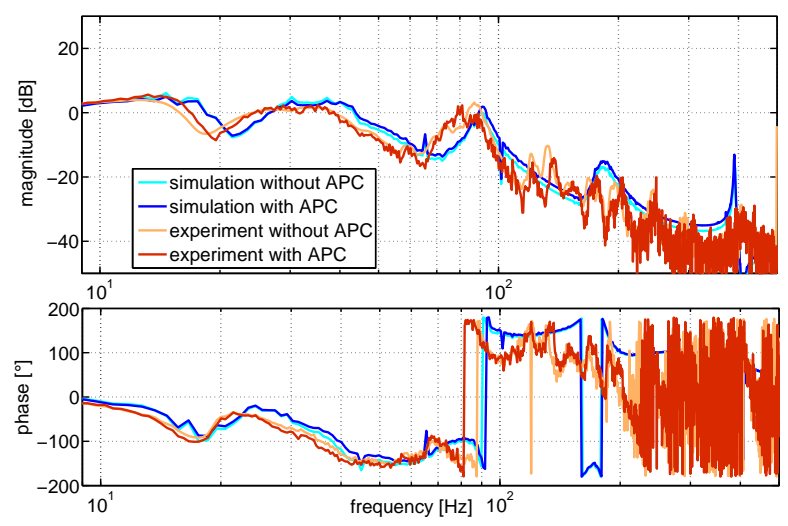

Fig. 14 Frequency response of simulation and experiment.

Furthermore, the same tendencies of using the APC option can be determined in the simulation and the experiment. Both step responses show a slightly better behavior with the APC option. Unfortunately, this improvement is relatively low, which can be referred to the low tuning factors which were used. It was not possible to use significantly higher tuning factors at the test rig because they caused an unstable system behavior which could not be predicted by the simulation.

In order to find the reasons for the deviations and the unstable system behavior, the frequency response is considered in Fig. 14. In general, both curve shapes of simulation and experiment show a similar trend. However, the lower frequency ranging up to about 80 $\mathrm{Hz}$ is not reproduced with the required accuracy, as it would be necessary for a more detailed investigation of the APC option. Because of the fact that especially the second dominating frequency of $30 \mathrm{~Hz}$ was lower than in the simulation, the positive damping effect of APC, predicted in the simulation, could not be reproduced at the test rig.

The lower frequencies were damped to a smaller extend then the amplifying of dominating frequencies above $80 \mathrm{~Hz}$. As a result, also rather small tuning factors of $K_{\mathrm{APC}}=1.0$ led to an almost unstable behavior because of the significant excitation at the dominating frequency of $93 \mathrm{~Hz}$.

\section{Summary and Outlook}

This paper is concerned with the improvement of the dynamic behavior of direct driven servo axes. Therefore, a ball screw test rig was analyzed, for which a structural extension of the standard PI velocity control structure is examined. The extension is characterized by an additional velocity feedback which has the potential to improve the controller performance significantly due to a better damping of low frequent vibrations.

For the investigation, a simulation model was created based on the measured frequency response of the controlled system. With this model, the effects of the APC tuning factors and an additional filter were discussed in the time and frequency domain. It turned out, that the tuning factors cause a damping of low frequent vibrations and an amplification of the following higher frequencies. This amplification is the critical point which moves the system behavior close to the stability limit. The investigation showed that the tuning factors have the potential to damp the lower frequent vibrations which limit the dynamic behavior of the control structure.

To find an optimal setting for the APC parameters, a simulation based optimization with the criterion of the Integral of Absolute Error was carried out. It revealed an optimal setting with $K_{\mathrm{APC}}=3.07$ and $T_{\mathrm{APC}}$ $=3.81 \mathrm{~m} / \mathrm{s}$ and reduced the IAE about two times. Furthermore, an additional filter was used to reduce the excitation of the dominating frequency at $90 \mathrm{~Hz}$. Due to this, the IAE could be reduced more than three 
times. In addition, the Prony analysis was carried out to verify the damping and amplification of the respective frequencies.

For the verification of the simulated results, experimental investigations at the test rig were performed. Therefore, the influences of the APC tuning factors could be reproduced at the test rig. It was stated that the amplitudes of the simulation and the experiment showed a good accordance.

Although they indicate the same tendency, a deviation of the simulation and the experiment was identified. The consideration in the frequency responses revealed that the lower frequencies up to about $80 \mathrm{~Hz}$ could not be reproduced with the required accuracy. Hence, the tuning factors of APC could not significantly be increased in the experiment because of an unstable system behavior.

To compensate these deviations in the simulation, the model of the controlled system has to be adjusted. Especially the reproduction of the lower frequencies has to be improved. In order to prevent the unstable system behavior, the influence of the additional filter in the APC feedback has to be considered in more detail. Due to the reduction of the excitation of the dominant frequency and raising the phase, an improvement of the control performance should be achieved.

\section{Acknowledgments}

This work was funded by the German Federal Ministry for Education and Research (BMBF), Project Number KF2232911RU2.

\section{References}

[1] Gruner, M., and Mauermann, R. 2009. “Advanced
Possibilities in the Application of Servo-Screw-Presses.” Presented at the Kongress Stanztechnik, Dortmund, Germany.

[2] Zirn, O., and Jaeger, C. 2010. "Vibration Damping for Machine Tool Servo Drives by Load Acceleration Feedback.” Presented at the 2010 IEEE International Symposium on Industrial Electronics, Bari.

[3] Muenster, R., Schoenherr, R., Schlegel, H., and Drossel, W. G. 2013. "Analysis of a Position Control Extension on the Model of a Servo-Screw-Press.” Presented at the 2013 International Symposium on Electrodynamic and Mechatronic System, Zawiercie.

[4] Siemens AG. 2008. SINAMICS S120/S150 User Manual.

[5] Vukosavic, S., and Stojic, M. 1988. "Suppression of Torsional Oscillations in a High-Performance Speed Servo Drive." IEEE Transactions on Industrial Electronics 45 (1): 108-17.

[6] Siemens AG. 2006. "APC-Advanced Position Control Option.” Simodrive User Manual.

[7] Schütte, F. 2003. "Automated Controller Commissioning for Electric Drives with Oscillating Mechanics.” Dissertation thesis, Shaker Verlag, Aachen.

[8] Schröder, D. 2008. Electric Drives-Control of Power Systems. Berlin-Heidelberg: Springer Verlag.

[9] Neugebauer, R., Hipp, K., Hellmich, A., and Schlegel, H. 2012. "Increased Performance of a Hybrid Optimizer for Simulation Based Controller Parameterization.” Journal of Automation, Mobile Robotics \& Intelligent Systems 6: 42-5.

[10] Hipp, K., Hellmich, A., Schlegel, H., and Drossel, W. G. 2013. "Assessment of Optimization Criteria in the Time Domain Used for Simulation Based Controller Parameterization.” Presented at the 2013 International Symposium on Electrodynamic and Mechatronic System, Zawiercie.

[11] Schönherr, R., Schlegel, H., and Drossel, W. G. 2012. Assessing the Performance of Servo Drive Feedback Controls. Linz: Trauner.

[12] Baracle, A., Carpinelli, G., Lauria, D., Leonowicz, Z. 2004. "On Some Spectrum Estimation Methods for Analysis of Nonstationary Signals in Power Systems.” In Proceedings of the International Conference on Harmonic and Quality of Power, 260-5. 